\title{
Cross-Cultural Validation of the 5-Item
}

\section{Compliance Questionnaire for Rheumatology to the Arabic Language in Patients with Rheumatoid Arthritis}

\author{
Mohammed A Omair (ID) \\ Fatima Al Suwayeh ${ }^{2}$ \\ Alanoud Almashaan ${ }^{2}$ \\ Eman Alqurtas' \\ Mohammed K Bedaiwi (D) \\ Ibrahim Almaghlouth' \\ Abdulaziz Alkahalaf' \\ Haya M Almalaq $\mathbb{D D}^{2}$ \\ 'Rheumatology Unit, Department of \\ Medicine, King Saud University, Riyadh, \\ Saudi Arabia; ${ }^{2}$ Clinical Pharmacy \\ Department, College of Pharmacy, King \\ Saud University, Riyadh, Saudi Arabia
}

Purpose: A simple measure to assess drug adherence in Saudi patients with rheumatoid arthritis (RA) is required. The aim of this study was to translate and validate the 5-Item Compliance Questionnaire for Rheumatology (CQR5) into Arabic.

Patients and Methods: The questionnaire was translated and culturally adapted to Arab patients in six steps: initial translation, synthesis of the translation, back translation, expert committee review, test of the pre-final version, and development of the Arabic CQR5 (ACQR-5). The resulting version was tested for validity in patients with RA.

Results: A total of 103 adult patients with RA were recruited from rheumatology clinics at a university hospital in Riyadh, Saudi Arabia. After extensive translation, the final tool (CQR) was piloted in 15 patients. The final validation was performed with 88 patients. Of these, $80(90.9 \%)$ were female and $43.2 \%$ were seropositive. The mean $( \pm \mathrm{SD})$ age and disease duration were $50( \pm 13)$ and $11.4( \pm 8.2)$ years, respectively. Cronbach's alpha reliability was 0.886 , and the Kaiser-Meyer-Olkin measure of sampling adequacy for factor analysis was $0.870(\mathrm{p}<0.001)$. The mean ACQR-5 was 17.78 (2.70), with 14 $(15.9 \%)$ classified as low adherents and the remaining $74(84.1 \%)$ as high adherents. Binary logistic regression revealed that increasing age (odds ratio [OR] 1.082, 95\% confidence interval $[\mathrm{CI}]$ : $1.025-1.142, \mathrm{p}=0.005)$ and a trend toward the presence of other comorbidities (OR 3.111, 95\% CI: 0.961-10.070, p=0.058) were associated with low adherence.

Conclusion: ACQR-5 is a simple and feasible tool for identifying adherence levels in patients with RA in Saudi Arabia. A high level of adherence was observed in this study. Additional studies are required to assess ACQR-5 validity and adherence levels in a larger, more diverse population.

Keywords: adherence, rheumatoid arthritis, compliance questionnaire for rheumatology, Saudi Arabia

\section{Plain Language Summary}

The 5-Item Compliance Questionnaire for Rheumatology has been developed and validated in the English language to assess drug adherence in patients with rheumatic diseases. The aim of the current study is to translate and validate the CQR to the Arabic language on a cohort of rheumatoid arthritis (RA) patients. The product of this study ACQR-5 is a simple and reliable tool to assess adherence in Arabic RA patients. Further studies are needed to assess its use in other disease areas.
Correspondence: Mohammed A Omair Rheumatology Division, Department of Medicine (38), College of Medicine King Saud University, PO Box 2925, Riyadh,

I I46I, Saudi Arabia

Tel/Fax+966-I-467-I546

Email momair@ksu.edu.sa 


\section{Introduction}

Rheumatoid arthritis (RA) is the most common form of chronic inflammatory arthritis and is associated with loss of function, reduced health-related quality of life (HRQOL), and premature mortality. ${ }^{1,2}$ Pharmacological treatment with disease-modifying anti-rheumatic drugs (DMARDs) is the mainstay line for the management of patients with RA according to regional and national guidelines. ${ }^{3,4}$ Adherence to medications is defined by the World Health Organization (WHO) as "the degree to which the person's behavior corresponds with the agreed recommendations from a health care provider". 5 Similar to other chronic illnesses, non-adherence in patients with RA is a major challenge in daily clinical practice, which is associated with increased health costs, poor treatment outcomes, increased risk of flare, and long-term disability. ${ }^{6}$ Adherence assessment can be performed indirectly through electronic records, pharmacy records, tablet counts, and questionnaires. The first three tools require a good health system or a pharmacist. Questionnaires are simple, self-administered, and inexpensive. All indirect tools do not actually prove drug administration. Direct assessment can be performed through measurements of drug levels or their metabolites. These levels are a more accurate measure of adherence, but are also more invasive, bear a higher cost, and can be affected by metabolic variation. ${ }^{7}$ Questionnaires are classified into generic such as the Medication Adherence Report Scale (MARS) ${ }^{8}$ or disease-specific such as the Compliance Questionnaire for Rheumatology (CQR). The CQR is the only tool that has been validated for patient with rheumatic. Its first version consisted of 19 items $^{9}$ and was subsequently modified and simplified to a five-item scale (CQR-5). ${ }^{10}$ The time to fill the CQR-5 is less than 2 minutes making it a simple and feasible to be incorporated in daily clinical practice. Previous studies have shown a high rate of nonadherence in Saudi patients with rheumatic diseases using generic tools. ${ }^{11}$ The aim of the current study was to translate the CQR-5 into Arabic (ACQR-5) and validate it with a sample of patients with RA.

\section{Patients and Methods}

\section{Patients}

This was a cross sectional study that involved patients with RA who fulfilled the American College of Rheumatology/European League Against Rheumatism (ACR/EULAR) 2010 classification criteria $^{12}$ with a disease duration of $\geq 6$ months on at least one DMARD therapy, were included in the study. Exclusion criteria were illiteracy, major mental illness, inability to complete the questionnaire, drug-free remission, and discontinuation of all RA-related therapies for a period of $\geq 4$ weeks for any reason. The questionnaire specifically asked about adherence to DMARDs. Methotrexate (MTX) was administered weekly, while other conventional synthetic DMARDs (csDMARDs) or targeted synthetic DMARDs (tsDMARDs) were administered daily or twice daily. The frequency of subcutaneous (SQ) and intravenous (IV) biological DMARD (bDMARD) use ranged from weekly (etanercept, SQ tocilizumab, and SQ abatacept), to biweekly (adalimumab), monthly (certolizumab, IV tocilizumab, IV abatacept, and golimumab), and every 6-8 weeks (infliximab). Patients receiving rituximab monotherapy were excluded from the study.

Patients were recruited from the RA-Specialized Rheumatology Clinics, which comprises a multidisciplinary team that includes a clinical pharmacist that delivers pharmaceutical care and assesses adherence and drug-related issues. Recruitment was initially planned to occur during clinic visits, but due to the coronavirus disease pandemic, an online survey was developed and sent between July 2020 and January 2021. Patients completed the form twice, at least two weeks apart, to minimize recall bias. This study was reported in accordance with the STROBE guidelines. ${ }^{13}$

\section{Stepwise Translation Process}

The author of the CQR5 was contacted and permission was obtained for the translation of the CQR5 into Arabic. A stepwise translation process was initiated, guided by Beaton et al's process for cross-cultural adaptation of selfreport measures. ${ }^{14}$ The CQR5 was forward translated by five Arabic speaking bilingual individuals with health care specialties. The five translations were reviewed by the research team, and discrepancies were resolved to create a single document. Thereafter, the translated document was sent to two rheumatologists for face validity, and it was amended based on their reviews. The final translated document was sent to a certified translation company for back-translation into the original English language. The back-translated English tool was compared with the original tool to determine whether there were any major discrepancies (Figure 1). No differences were found when compared to the original tool. Finally, to check the clarity of the translated Arabic tool, a pilot study was carried out 


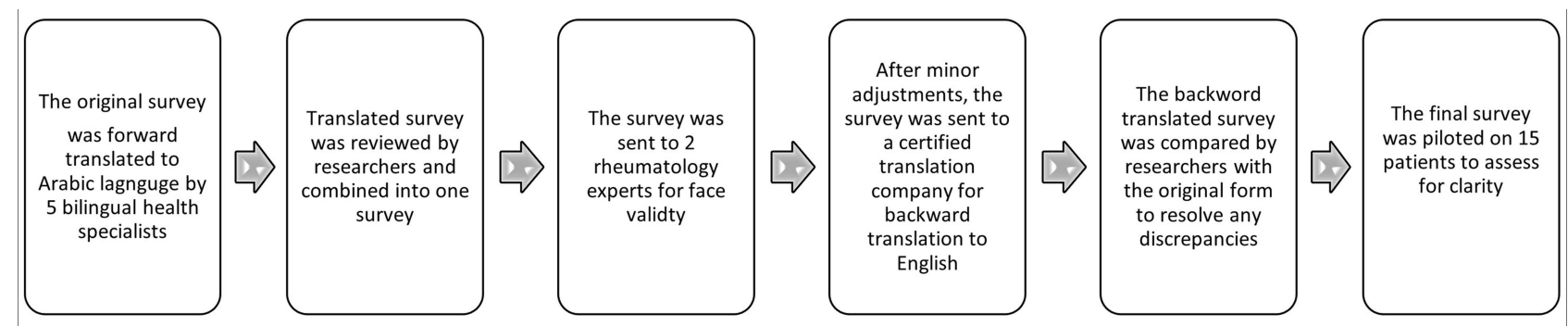

Figure I Step-by-step process for survey translation.

through interviews with 15 participants to review and comment on the tool clarity. To calculate test-retest reliability, the survey was readministered to the same participants, by the same researchers, a minimum of two weeks from the date of the first survey.

\section{Tools and Measurements}

The English language validated CQR5 is a selfadministered survey with the purpose of identifying the adherence of patients with RA. The CQR5 score ranged from (scored 1) "Definitely don't agree" to (scored 4) "Definitely agree" where patients with higher scores were considered to have high adherence. ${ }^{10}$ The score was computed as high or low adherence based on an equation provided by the author of the original work. In addition to the CQR5, we collected information on demographics, marital status, living status, comorbidities, medications, and the Arabic validated Health Assessment Questionnaire Disability Index (HAQ-DI). ${ }^{15}$ The HAQDI is a self-reported questionnaire covering 20 items in eight domains that measure difficulty in performing activities of daily living: dressing, arising, eating, walking, hygiene, reach, grip, and common daily activities. Each question was rated on a $0-3$ scale, where 0 indicated "without difficulty" and 3 indicated "unable to do"; additional points could be added if aids or devices were needed for specific activities. Increasing scores indicated decreasing functionality. In each domain, the higher score was assigned as a score of the entire domain. A total of eight scores were combined and divided by 8 to give a final score ranging from 0 to 3 . A score of $0.0-1.0$ indicated mild disability, 1.1-2.0 was moderate, and 2.1-3.0 was high.

\section{Statistical Analysis}

Data were coded, entered, and statistical analyses were performed using the Statistical Package for Social
Sciences (SPSS) version 26. Each patient was assigned a unique study number to ensure complete patient confidentiality. Descriptive statistics (means, SD, counts, and percentages) were used to describe the subjects' demographics. Reliability was checked using Cronbach's alpha, and the questionnaire was applied twice, with a 2 -week interval. The intraclass correlation coefficient was calculated to assess test-retest reliability and to determine whether there were differences between the first and second CQR5. The Kaiser-Meyer-Olkin test was performed to evaluate sample adequacy for factor analysis. Principle factor analysis was conducted to confirm that the survey had a single construct, and the number of components was determined according to the eigenvalue and oblique (direct) rotation. Included patients were then classified as having high or low adherence after computing the CQR5 using the CQR calculator. Binary logistic regression was applied to compute the odds ratio (OR) of having low or high adherence with the resulting $\mathrm{p}$ value and $95 \%$ confidence intervals (CI).

\section{Ethical Considerations}

This study was conducted in accordance with the Declaration of Helsinki. Ethical approval was granted by the institutional review board of King Saud University and the medical city project number E-20-4913. All patients were informed about the purpose of the study and agreed to participate and provided their electronic consent prior to study conception.

\section{Results}

\section{Reliability Testing}

Different reliability tests were conducted for the survey, with a Cronbach's alpha reliability coefficient of 0.886 . The intraclass correlation coefficient for test-retest of the survey was 0.870 ( $\mathrm{p}<0.001)$. Factor analysis was based on the Kaiser-Meyer-Olkin measure of sampling adequacy which 
Table I Arabic CQR-5 Survey Reliability Test Results

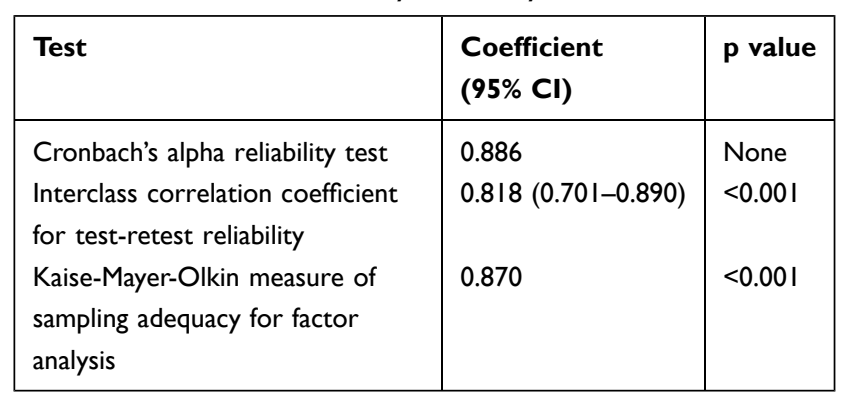

Abbreviation: $\mathrm{Cl}$, confidence interval.

showed a value of $0.870(\mathrm{p}<0.001)$. In the correlation analysis performed for the test-retest reliability, an adequate, statistically significant, and positive correlation was observed between the first test and the retest scores with a correlation coefficient of $0.818(0.701-0.89095 \% \mathrm{CI}$, $\mathrm{p}<0.001$ ) (Table 1). In factor analysis, we found that only one component was available, with an eigenvalue of 3.494, which is greater than one and, therefore, higher than average (Figure 2). All items were strongly correlated, showing that the survey assessed one construct (compliance).

\section{Demographics and Compliance Scores}

A total of 103 adult patients with RA were recruited to participate in the study. The survey instrument was piloted with 15 patients. Then the final validated form was undertaken with 88 patients. Of those, 80 (90.9\%) were female, with a mean (standard deviation) age and disease duration of $50( \pm 13)$ and $11.4( \pm 8.2)$ years, respectively. Eighty-four (95.5\%) were Saudis, 60 (68.2\%) were living in Riyadh,
$64(72.7 \%)$ were married, 60 (68.2\%) were unemployed, and $85(96.6 \%)$ had never smoked. Sixty-three patients $(71.6 \%)$ had at least one comorbidity, and 38 (43.2\%) were seropositive. Background medications were as follows: 46 (52.3\%) were on csDMARDs with methotrexate (MTX) being the most common, 25 (28.4\%) patients were on bDMARDs. Of those patients, $22(27.2 \%)$ were on a combination of bDMARDs and csDMARDs. Concomitant prednisolone was used by $17(19.3 \%)$ patients with a mean dose of 7.53 $\pm 3.5 \mathrm{mg}$ /day. The majority, 52 (59.1\%), had mild disability with a mean \pm SD of $0.946 \pm 0.745$ and a pain score of $5 \pm 3$ on a 10 -Likert scale (Table 2).

The mean ACQR-5 score was 17.78 (2.70), with 14 (15.9\%) classified as low compliance and the remaining 74 (84.1\%) as high compliance. While exploring average responses per question using a radar chart, the most influential variable on adherence, in total, was "I take my medicines because I have complete trust in my rheumatologist", and the lowest adherence was due to a lack of confidence in the medication with a low response to the statement "I definitely don't dare to miss my anti-rheumatic medicines" (Figure 3).

\section{Compliance Factors}

Binary logistic regression revealed that increasing age (OR $1.082,95 \% \mathrm{CI}: 1.025-1.142, \mathrm{p}=0.005)$ and a trend toward the presence of other comorbidities (OR 3.111, 95\% CI: 0.961$10.070, \mathrm{p}=0.058$ ) was associated with low compliance but was not significant. In addition, HAQ-DI was increased in non-compliant individuals with a non-adjusted OR of 2.874 (95\% CI 1.058-7.808, $\mathrm{p}=0.038)$. Using univariate analysis,

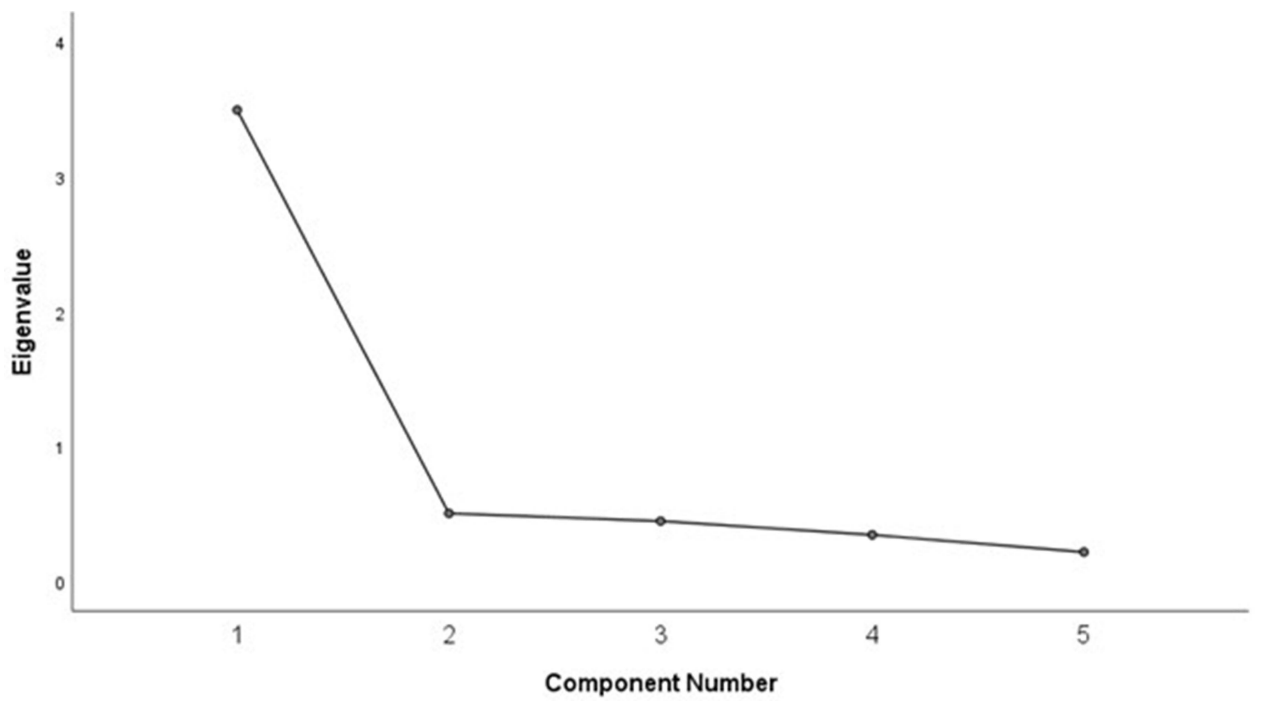

Figure 2 Factor analysis of survey components: Arabic CQR5. 
Table 2 Baseline Characteristics of the Study Population $(n=88)$ Arabic CQR5

\begin{tabular}{|c|c|c|}
\hline \multicolumn{2}{|l|}{ Characteristics of 88 Participants } & \multirow{2}{*}{$\begin{array}{c}\mathbf{N}(\%) \\
78(92.9)\end{array}$} \\
\hline Care giver or patient & Patient & \\
\hline Gender & Female & $80(90.9)$ \\
\hline Age, years mean (SD) & \multicolumn{2}{|c|}{$50(13)$} \\
\hline Nationality & Saudi & $84(95.5)$ \\
\hline Living & In Riyadh & $60(68.2)$ \\
\hline Marital & $\begin{array}{l}\text { Divorced } \\
\text { Single } \\
\text { Widowed } \\
\text { Married }\end{array}$ & $\begin{array}{c}6(6.8) \\
8(9.1) \\
10(11.4) \\
64(72.7)\end{array}$ \\
\hline Occupation & Unemployed & $60(68.2)$ \\
\hline Smoking Status & $\begin{array}{l}\text { Previous } \\
\text { smoker } \\
\text { Smoker } \\
\text { Never } \\
\text { smoked }\end{array}$ & $\begin{array}{c}2(2.3) \\
1(1.1) \\
85(96.6)\end{array}$ \\
\hline Presence of Other comorbidities & Yes & $63(71.6)$ \\
\hline $\begin{array}{l}\text { Heath assessment questionnaire and } \\
\text { disability index, HAQ-DI }\end{array}$ & $\begin{array}{l}0.0-1.0 \text { Mild } \\
1.1-2.0 \\
\text { Moderate } \\
2.1-3.0 \text { severe }\end{array}$ & $\begin{array}{c}52(59.1) \\
28(31.8) \\
8(9.1)\end{array}$ \\
\hline HAQ-DI, mean (SD) & \multicolumn{2}{|c|}{$0.946(0.745)$} \\
\hline Pain-0-10, mean (SD) & \multicolumn{2}{|c|}{$5(3)$} \\
\hline CQR total score, mean (SD) & \multicolumn{2}{|c|}{$17.78(2.70)$} \\
\hline CQR score & $\begin{array}{l}\text { Low } \\
\text { adherence } \\
\text { High } \\
\text { adherence }\end{array}$ & $\begin{array}{l}14(15.9) \\
74(84.1)\end{array}$ \\
\hline Corticosteroid use & Yes & $17(19.3 \%)$ \\
\hline bDMARDs & Yes & $25(28.4)$ \\
\hline csDMARDs & Yes & $46(52.3)$ \\
\hline
\end{tabular}

Abbreviations: CQR, Compliance Questionnaire for Rheumatology; HAQ-DI, Health Assessment Questionnaire Disability Index; bDMARDs, Biologic Disease Modifying Anti-Rheumatic Drugs, csDMARDs; Conventional Synthetic Disease Modifying Anti-Rheumatic Drug.

only age and education were significantly different; therefore, they were entered into the regression model. The resulting significant, adjusted OR of the HAQ-DI was lost, indicating that it was affected by age, education, or both, with an adjusted odds ratio of 1.815 (95\% CI $0.591-5.573$, $\mathrm{p}=0.298$ ). Data of unadjusted and adjusted ORs, exploring factors related to CQR scores, are available in Table 3.

\section{Discussion}

This study developed and validated a feasible method to rapidly assess drug compliance in Saudi patients, which can lead to high persistence. Medication non-compliance is a common and complex challenge in managing patients with RA globally. While compliance and persistence are intricately connected, they do not reflect the same thing. Heterogeneity in assessing these two measures has been the main obstacle in performing meta-analyses in systematic reviews, resulting in a wide range of results. In 2013, Fidder et al performed a systematic review on compliance with adalimumab, etanercept, and infliximab in patients with inflammatory bowel disease (IBD) and patients with RA. The pooled compliance was 59\% (95\% CI: 58-60\%) in patients with RA compared to 70\% (95\% CI: 67-73\%) in patients with IBD. ${ }^{16}$ This was followed by another systematic review in 2018, on the same drugs that revealed a mean compliance level of $63 \%$ for both etanercept and adalimumab and $54 \%$ for infliximab. ${ }^{17}$ Curtis et al reported the persistence of MTX to be in the range of $50-94 \%$ at 1 year, dropping to $25-79 \%$ at 5 years. ${ }^{18}$

The CQR was specifically developed in patients with rheumatic diseases. The first version was validated against electronic medication event monitoring. 9 Subsequently, a 5 -item version was developed. ${ }^{10}$ This tool has been validated in different languages, including Italian, Spanish, ${ }^{19}$ Hindi, $^{20}$ Turkish $^{21}$ and Korean. ${ }^{22}$

The high compliance rate in the current study might be related to the small sample size or a real observation due to the structure of the Specialized Rheumatology Clinic that includes pharmaceutical care in the form of a pharmacist who counsels patients on adherence, medication administration, and adverse events. A survey of the members of the Saudi Society for Rheumatology was conducted in 2015. When asked about the factors that most influence treatment selection; route of administration (70\%), evidence available in the literature (61\%), underlying comorbidities (61\%), and patient choice (59\%) were the most selected options. ${ }^{23}$ This behavior, of engaging patients in their treatment decisions, might improve treatment-related outcomes and compliance. ${ }^{24}$

The validated questionnaire showed good reliability and can be regularly administered in daily clinical practice that will lead to better assessment and drug utilization. Additionally, the inputs of low and high compliance can be easily interpreted by clinicians and communicated to patients and their caregivers. Additionally, the availability 


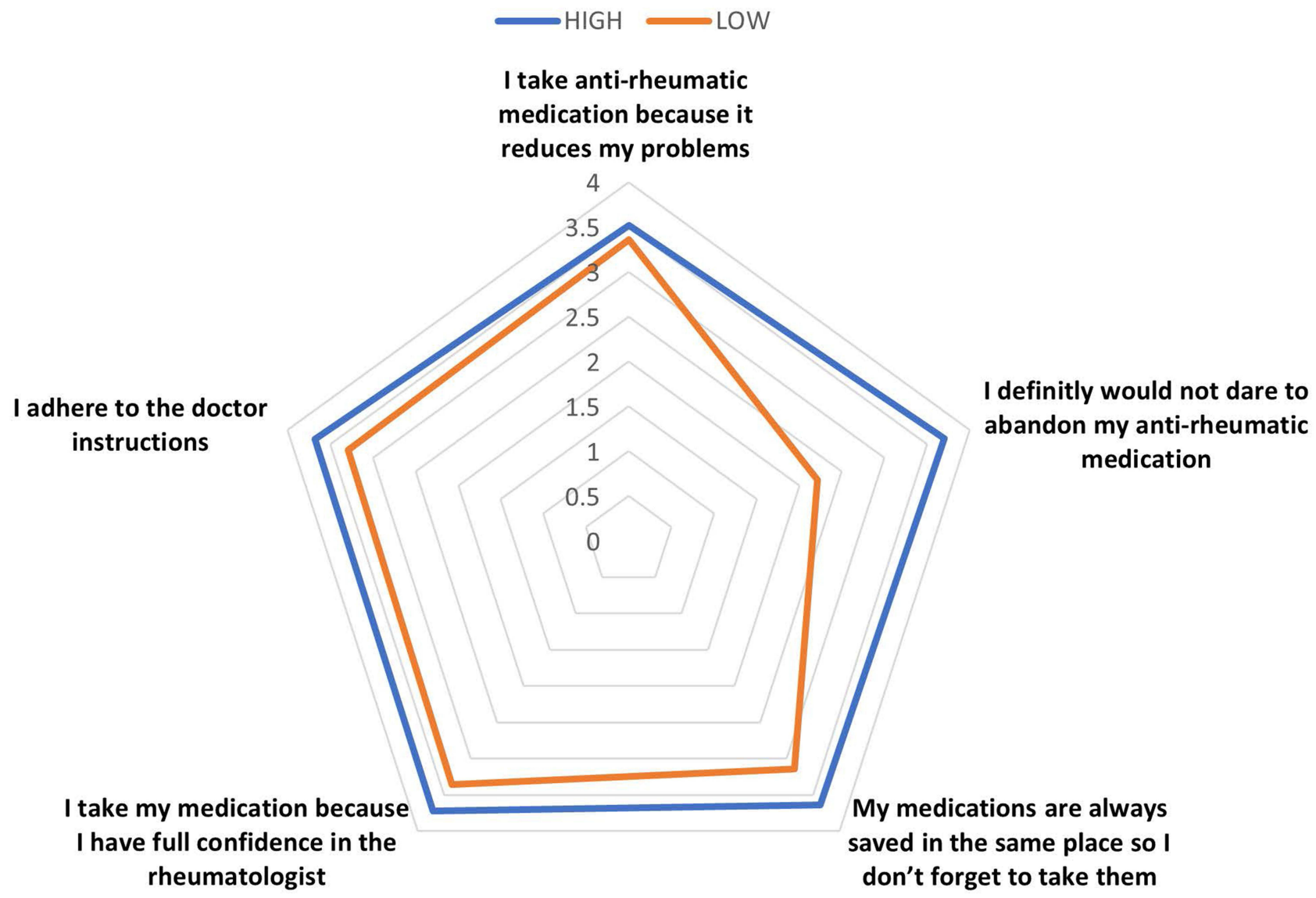

Figure 3 Radar chart showing by low and high adherence according to CQR score: Arabic CQR5

of ACQR-5 will allow researchers to explore compliance and compliance related issues in observational and interventional studies.

Table 3 Binary Logistic Regression of Factors Affecting Adherence According to Compliance Questionnaire for Rheumatology Low or High Outcome with High Adherence as the Reference Group

\begin{tabular}{|l|c|c|c|}
\hline & $\begin{array}{c}\text { Odds } \\
\text { Ratio }\end{array}$ & $\begin{array}{c}\text { 95\% Confidence } \\
\text { Intervals }\end{array}$ & P value \\
\hline Gender, females & 0.529 & $0.095-2.939$ & 0.467 \\
Age, Years & 1.082 & $1.025-1.142$ & $0.005^{*}$ \\
Living, Riyadh & 1.232 & $0.37 I-4.086$ & 0.733 \\
Occupation, employed & 2.524 & $0.789-8.076$ & 0.119 \\
Married, Yes & 0.688 & $0.174-2.717$ & 0.594 \\
Education, Low & 0.142 & $0.018-1.147$ & 0.067 \\
Other comorbidities, Yes & 3.111 & $0.961-10.070$ & 0.058 \\
Corticosteroid, Yes & 2.106 & $0.540-8.219$ & 0.284 \\
Convectional synthetic & 0.792 & $0.250-2.506$ & 0.691 \\
DMARDs, Yes & & & \\
Biologic DMARDs, Yes & 2.706 & $0.560-13.082$ & 0.216 \\
\hline
\end{tabular}

Note: *Statistically significant.

Abbreviation: DMARDs, Disease Modifying Anti-Rheumatic Drugs.
Limitations of the current study include the small sample size and lack of disease-related outcome measures, nonspecific assessment of questionnaires on all DMARDs. The lack of external validity to other established compliance tools due to the unavailability in Arabic or copyright restrictions. Comparators such as the pill count and pharmacy refill were difficult to apply due to the COVID-19 pandemic. As outpatient visits were limited and pharmacy refill was substituted by medication home delivery.

The low representation of specific demographics such as male sex, early disease, and non-Saudis need to be taken in consideration in future studies. Currently, a nationwide study on compliance in Saudi patients with RA, has been launched.

\section{Conclusion}

In conclusion, the current study provides a validated Arabic tool that can be used to assess treatment compliance in patients with RA and other rheumatic diseases. Further studies are needed to confirm its external validity and evaluate the response to interventions. 


\section{Disclosure}

All authors disclose no relevant conflicts of interest related to this work.

\section{References}

1. Norton S, Fu B, Scott DL, et al. Health assessment questionnaire disability progression in early rheumatoid arthritis: systematic review and analysis of two inception cohorts. Semin Arthritis Rheum. 2014;44(2):131-144. doi:10.1016/j.semarthrit.2014.05.003

2. Omair MA, Erdogan A, Tietz N, Alten R. Physical and emotional burden of rheumatoid arthritis in Saudi Arabia: an Exploratory Cross-Sectional Study. Open Access Rheumatol. 2020;12:337-345. doi:10.2147/OARRR.S284734

3. Smolen JS, Landewé RBM, Bijlsma JWJ, et al. EULAR recommendations for the management of rheumatoid arthritis with synthetic and biological disease-modifying antirheumatic drugs: 2019 update. Ann Rheum Dis. 2020;79(6):685-699. doi:10.1136/annrheumdis-2019216655

4. Arayssi T, Harfouche M, Darzi A, et al. Recommendations for the management of rheumatoid arthritis in the Eastern Mediterranean region: an adolopment of the 2015 American College of Rheumatology guidelines. Clin Rheumatol. 2018;37(11):2947-2959. doi:10.1007/s10067-018-4245-5

5. Jimmy B, Jose J. Patient medication adherence: measures in daily practice. Oman Med J. 2011;26(3):155-159. doi:10.5001/omj.2011.38

6. Li L, Cui Y, Yin R, et al. Medication adherence has an impact on disease activity in rheumatoid arthritis: a systematic review and meta-analysis. Patient Prefer Adherence. 2017;11:1343-1356. doi:10.2147/PPA.S140457

7. Van Den Bemt BJ, Zwikker HE, Van Den Ende CH. Medication adherence in patients with rheumatoid arthritis: a critical appraisal of the existing literature. Expert Rev Clin Immunol. 2012;8 (4):337-351. doi:10.1586/eci.12.23

8. Cohen JL, Mann DM, Wisnivesky JP, et al. Assessing the validity of self-reported medication adherence among inner-city asthmatic adults: the Medication Adherence Report Scale for Asthma. Ann Allergy Asthma Immunol. 2009;103(4):325-331. doi:10.1016/S10811206(10)60532-7

9. de Klerk E, van der Heijde D, Landewe R, van der Tempel H, van der Linden S. The compliance-questionnaire-rheumatology compared with electronic medication event monitoring: a validation study. J Rheumatol. 2003;30(11):2469-2475.

10. Hughes LD, Done J, Young A. A 5 item version of the Compliance Questionnaire for Rheumatology (CQR5) successfully identifies low adherence to DMARDs. BMC Musculoskelet Disord. 2013;14:286. doi:10.1186/1471-2474-14-286

11. Alsowaida N, Alrasheed M, Mayet A, Alsuwaida A, Omair MA. Medication adherence, depression and disease activity among patients with systemic lupus erythematosus. Lupus. 2018;27 (2):327-332. doi:10.1177/0961203317725585
12. Aletaha D, Neogi T, Silman AJ, et al. 2010 Rheumatoid arthritis classification criteria: an American College of Rheumatology/ European League Against Rheumatism collaborative initiative. Arthritis Rheum. 2010;62(9):2569-2581. doi:10.1002/art.27584

13. von Elm E, Altman DG, Egger M, et al. The Strengthening the Reporting of Observational Studies in Epidemiology (STROBE) statement: guidelines for reporting observational studies. $J$ Clin Epidemiol. 2008;61(4):344-349. doi:10.1016/j.jclinepi.2007.11.008

14. Beaton DE, Bombardier C, Guillemin F, Ferraz MB. Guidelines for the process of cross-cultural adaptation of self-report measures. Spine. 2000;25(24):3186-3191. doi:10.1097/00007632-20001215000014

15. El Meidany YM, El Gaafary MM, Ahmed I. Cross-cultural adaptation and validation of an Arabic Health Assessment Questionnaire for use in rheumatoid arthritis patients. Joint Bone Spine. 2003;70 (3):195-202. doi:10.1016/S1297-319X(03)00004-6

16. Fidder HH, Singendonk MM, van der Have M, Oldenburg B, van Oijen MG. Low rates of adherence for tumor necrosis factor-alpha inhibitors in Crohn's disease and rheumatoid arthritis: results of a systematic review. World $J$ Gastroenterol. 2013;19 (27):4344-4350. doi:10.3748/wjg.v19.i27.4344

17. Murage MJ, Tongbram V, Feldman SR, et al. Medication adherence and persistence in patients with rheumatoid arthritis, psoriasis, and psoriatic arthritis: a systematic literature review. Patient Prefer Adherence. 2018;12:1483-1503. doi:10.2147/PPA.S167508

18. Curtis JR, Bykerk VP, Aassi M, Schiff M. Adherence and persistence with methotrexate in rheumatoid arthritis: a systematic review. J Rheumatol. 2016;43(11):1997-2009. doi:10.3899/jrheum.151212

19. Salgado E, Maneiro Fernández JR, Souto Vilas A, Gómez-Reino JJ. Spanish transcultural adaptation and validation of the English version of the compliance questionnaire in rheumatology. Rheumatol Int. 2018;38(3):467-472. doi:10.1007/s00296-018-3930-7

20. Shetty R, Reddy K, Inam S, Khera K. Impact of medication adherence by using Indian Version Compliance Questionnaire Rheumatology (Cqr) and Medication Adherence Report Scale (Mars) tools on quality of life of patients with rheumatoid arthritis. Value Health. 2014;17(7):A385. doi:10.1016/j.jval.2014.08.2639

21. Cinar FI, Cinar M, Yilmaz S, et al. Cross-cultural adaptation, reliability, and validity of the Turkish version of the compliance questionnaire on rheumatology in patients with Behcet's disease. J Transcult Nurs. 2016;27(5):480-486. doi:10.1177/1043659615577699

22. Lee JY, Lee SY, Hahn HJ, Son IJ, Hahn SG, Lee EB. Cultural adaptation of a compliance questionnaire for patients with rheumatoid arthritis to a Korean version. Korean J Intern Med. 2011;26 (1):28-33. doi:10.3904/kjim.2011.26.1.28

23. Omair MA, Omair MA, Halabi H. Survey on management strategies of rheumatoid arthritis in Saudi Arabia: a Saudi Society for Rheumatology Initiative. Int J Rheum Dis. 2017;20(9):1185-1192. doi:10.1111/1756-185X.12735

24. Marengo MF, Suarez-Almazor ME. Improving treatment adherence in patients with rheumatoid arthritis: what are the options? Int $J$ Clin Rheumtol. 2015;10(5):345-356. doi:10.2217/ijr.15.39
Patient Preference and Adherence

\section{Publish your work in this journal}

Patient Preference and Adherence is an international, peer-reviewed, open access journal that focusing on the growing importance of patient preference and adherence throughout the therapeutic continuum. Patient satisfaction, acceptability, quality of life, compliance, persistence and their role in developing new therapeutic modalities and compounds to optimize clinical outcomes for existing disease states are major areas of interest for the journal. This journal has been accepted for indexing on PubMed Central. The manuscript management system is completely online and includes a very quick and fair peer-review system, which is all easy to use. Visit http:// www.dovepress.com/testimonials.php to read real quotes from published authors. 